Review

\title{
Effects of Circadian Rhythm on Migraine Therapy
}

Yulia Salamatova $\mathrm{DO}^{1}$, Andreja Packard $\mathrm{MD}, \mathrm{PhD}^{2, *}$

1. Department of Neurology, University of Florida College of Medicine, US; E-Mail: yulia.salamatova@jax.ufl.edu

2. Neurology Department, Sleep Section, Arnold 22435 G, 1 South Prospect St, Burlington VT, US; E-Mail: andreja.packard@uvmhealth.org

* Correspondence: Andreja Packard; E-Mail: andreja.packard@uvmhealth.org

Academic Editor: Yasushi Shibata

Special Issue: The Pathophysiology and Treatment for Migraine

OBM Neurobiology

2021, volume 5, issue 4

doi:10.21926/obm.neurobiol.2104111
Received: June 24, 2021

Accepted: December 12, 2021

Published: December 20, 2021

\begin{abstract}
Circadian rhythms are 24-hour cycles of physical, mental, and behavioral changes regulated and maintained by the internal primary circadian clock, however modifiable by a number of external cues or "zeitgebers", the most powerful one being light. Core set of clock genes regulate the whole-body metabolism and transcription of over $40 \%$ of mammalian RNA, including that for drug transporters, binding and metabolizing proteins responsible for regulation of pharmacokinetics of vast array of medications. Growing amount of evidence also shows circadian rhythmicity of a number of patho-physiological processes, such as are migraine, chronic pain, and epilepsy, suggesting amenability to chronotherapy. Chronotherapy involves behavioral and pharmacological strategies to restore or correct illfunctioning circadian rhythm as well as manipulation of standardized treatments throughout the day to maximize therapeutic and minimize side effects, termed chronopharmacology. Chronotherapy for chronic migraines and headache variants using synchronization techniques as well as chronopharmacology of abortive and preventive migraine medications is being actively researched. In this review, we summarize current state of chronotherapy for
\end{abstract}

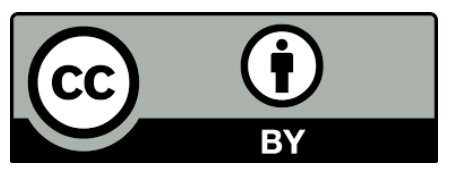

(C) 2021 by the author. This is an open access article distributed under the conditions of the Creative Commons by Attribution License, which permits unrestricted use, distribution, and reproduction in any medium or format, provided the original work is correctly cited. 
headache variants and discuss future prospects in circadian optimization of migraine headaches treatment.

\section{Keywords}

Migraine; headache; sleep; circadian rhythm; chronotherapy; chronopharmacology

\section{Biology of Mammalian Circadian Rhythm}

Circadian rhythms are 24-hour cycles of physiological, behavioral and cognitive changes which are entrained to the environment by external cues or "zeitgebers", but persist independently in the absence of external signals in almost all organisms [1]. They regulate the array of physiological processes, such as sleep/wake cycles, body temperature, hunger/satiety, blood pressure, heart rate, respiration, and pain modulation [1-7]. In mammals, circadian rhythms are regulated and maintained by the internal primary circadian clock, or "master clock", located in the suprachiasmatic nuclei (SCN) of ventral hypothalamus. The SCN coordinates peripheral clocks of body organs and individual cells to achieve maximum effectiveness, either in a direct manner (through SCN neurotransmitter signaling) or indirectly by controlling hormonal and autonomic outputs [8]. Optimal orchestration is then achieved by the ability of the SCN to reset in response to a number of environmental zeitgebers. The dominant synchronizing zeitgeber is light, likely due to the universal presence of cycles of light and dark on the Earth. Light activates melanopsin receptors on retinal ganglion cells, which then send activating glutamatergic signal via the retinohypothalamic tract directly to SCN neurons [9].

Intracellular circadian clock machinery depends on the transcription and translation of the core clock genes that can be thought of in three main groups. The first group of molecular clock proteins include CLOCK /BMAL1 dimers that are transcription factors governing transcription of downstream clock genes. The second group, that includes PER-CRY and REV-ERBs/RORs dimers, has the property to reenter the nucleus and inhibit CLOCK/BMAL1 in negative feedback loop $[10,11]$. The third clock protein group are essentially modifiers of the circadian rhythm that are able to speed up degradation or slow accumulation of the other clock proteins mostly via phosphorylation/dephosphorylation [12, 13]. This cascade of clock proteins effects up to $43 \%$ of mammalian RNA protein coding genes, which in turn have been shown to cycle in their abundance in one or more organs, typically in organ specific manner [14].

\section{Circadian Variation of Pain and Migraines}

Circadian rhythm changes in pain perception have been well established by numerous studies for a spectrum of pain conditions including migraines [15-17]. Clinically, symptoms of migraines and other types of primary headaches do cycle in patients, usually with different periodicities [18]. Migraine attacks mostly peak early in the morning or late at night, although some studies report an afternoon or biphasic peaks, likely related to the individual patient chronotypes [19]. Migraineurs more often belong to early chronotype group and most migraine attacks happen in the early mornings [20]. Circadian rhythms were shown to play an important role in inflammatory pathways, which are favored by current data as the main pathophysiological mechanisms of migraine [21, 22]. 
Circadian control of pain and neuroinflammation has recently been well covered in literature offering extensive lists of immune mediators and receptors whose expression is under the control of circadian genes [21]. For example, cytokines TNF- $\alpha$, IL-2, IFN- $\gamma$ and IL-10 peak during the resting period, while circadian misalignment results in increase of TNF- $\alpha, C$-reactive protein (CRP) and interleukin 10 (IL-10) [23]. In migraine patients, TNF- $\alpha$, CRP as well as neutrophil/monocyte and platelet/lymphocyte ratios (NMR and PLR) are higher compared to healthy controls [24]. In a murine model of chronic pain circadian rhythmicity of pain behavior and expression of pain-related NMDA receptor subunit and CREB in the SCN being the highest at the beginning of the active period [25]. Clock gene Per1 in spinal dorsal horn may play a crucial role in sensitivity to neuropathic pain as its downregulation leads to increase in mechanical sensitivity in mice [26].

Migraine process involves sensitization of the trigeminovascular system and activation of the hypothalamus which is central for sleep-wake cycle regulation, and stabilization of the arousal system of the brain $[22,27,28]$. Lateral hypothalamic peptidergic neurons produce orexin during wakefulness and melanin-concentrating hormone during REM sleep, thus augmenting inputs from brainstem to the cortex [27]. Interestingly, pituitary adenylate cyclase-activating peptide (PACAP) is a hypothalamic signaling molecule that plays a role in circadian clock, and is involved in pathophysiology of migraines typically being both elevated in patients with migraines and acting as a migraine trigger [28-30].

Circadian misalignment and sleep disturbances can increase migraine frequency [31, 32]. For example, familial migraine with aura in patients suffering from familial advanced sleep phase syndrome (FASPS) is associated with mutations in a serine-threonine kinase, casein kinase I delta $(C K I \delta)$, which reduce CKI $\delta$ activity and thus lead to reduced phosphorylation of Per2 and its greater stability [33]. Two specific mutations in the gene encoding CKI $\delta$ (T44A and H46R) were identified as associated with migraine phenotype. Mice carrying CKI $\delta$-T44A allele were more sensitive to pain, suggesting that decrease in CKI $\delta$ activity can contribute to the pathogenesis of migraine [34]. Melatonin, hormone secreted by the pituitary gland with control of hypothalamus has been extensively studied in headache. Specifically, melatonin levels are more sensitive to light exposure in familial migraine patients, thus suggesting higher light sensitivity in migraineurs not only during attacks, but also in between the episodes [35, 36]. Prolonged exposure to light in the Arctic population during the summer season correlates with more frequent headaches in migraine patients [37]. Nocturnal serum melatonin and urine metabolite levels are lower in adult patients suffering from migraine compared to healthy adults, with no difference after exclusion of patients with insomnia and depression [36]. $3 \mathrm{mg}$ of immediate release melatonin was found to be superior to placebo and comparable to nightly amitriptyline $25 \mathrm{mg}$ in decreasing migraine frequency [38-40]. In addition to proposed direct effects of melatonin concentration on migraines decreased availability of melatonin and a relative increase of $\mathrm{N}$-acetylserotoin/melatonin ratio in a setting of gut dysbiosis increase glutamatergic excitatory transmission in migraine [41].

Other zeitgebers, such as food intake and exercise, have also been noted to affect migraines. Stress and food deprivation are two frequently reported triggers of the migraine attacks. Prolonged fasting causes worsening of primary headaches, including migraines [42]. Missing meals has been self-reported as a migraine trigger by $57 \%$ of patients [43]. However premonitory migraine symptoms, such as decreased appetite and light sensitivity can overlap with migraine triggers and lead to skipping meals and light exposure being over reported in patient surveys. [44]. Interestingly night time snacking was shown to be associated with $40 \%$ decrease in migraine frequency compared 
to having no food [45]. The relationship between skipping meals and migraines is unclear, one of the proposed causes is hypoglycemia causing worsening of migraine [43].

While there are some observations that exercise can trigger migraine attacks by resetting circadian clock and sleep cycle, there is also evidence that regular exercise may have a prophylactic effect [46]. Timing of physical exercises was shown to cause circadian phase shifting by Youngstedt et al; they measured changes in the phase of melatonin metabolite 6-sulphatoxymelatonin (aMT6s) after subjects performed one-hour moderate treadmill exercises at different times of the day and demonstrated that morning and evening exercises produce a similar circadian clock resetting effect as exposure to light. The largest circadian phase delays were after exercising performed between 19:00 to 22:00 and the largest circadian phase advances at both 7:00 and from 13:00 to 16:00 [47]. Recent study of active students demonstrated protective effect of sport in female migraineurs, although the timing of the sports activity was not analyzed [48]. The proposed common neuromodulators in migraine and exercise are: calcitonin gene-related peptide (CGRP) increased in both exercising and migraine, endogenous opioids increased in exercise and decreased in headache and brain-derived neurotrophic factor (BDNF) elevated during migraine attacks and after exercise [49]. The relationship between migraine headache and exercising is rather complex and likely depends on multiple factors, such as the timing of exercise, intensity, duration and current presence of the migraine.

\section{Abortive Migraine Management and Circadian Rhythm}

Chronotherapy is defined as a precise timing of different therapeutic modalities including pharmacotherapy throughout the day to match body rhythms, optimize therapeutic outcomes and minimize side effects [50]. Specifically in migraine treatment, the timing of the ingestion of an abortive migraine medication can be predicted to result in different treatment outcomes spanning from ineffectiveness to toxicity as gastrointestinal absorption, liver and kidney metabolisms including drug degradation and excretion demonstrate strong circadian rhythmicity [50-55]. Absorption and pharmacokinetics of some migraine medications (e.g. indomethacin, nifedipine, valproic acid (VPA)) are time-dependent, their metabolism being affected by oscillations in expression of drug transporters, binding and metabolizing proteins [50, 56-58]. Indomethacin reaches its peak concentrations faster and peaks higher when taken before noon, compared to afternoon and evening injections [57]. VPA, which is often used in emergent/urgent settings as a onetime infusion of $500 \mathrm{mg}$ to abort migraine attack, also demonstrates circadian variability of absorption and metabolism demonstrating higher blood concentration after the morning dose compared to the evening dose [59]. Furthermore, VPA was shown to upregulate the expression of circadian clock genes encoding core circadian transcription factors regulating both positive and negative limbs of feedback loop [60]. Caffeine as the adenosine receptor blocker has different activity during the period of wakefulness, being most effective in decreasing migraine pain in the afternoon hours [61]. Chronopharmacokinetics of sumatriptan studied in a small group of volunteers demonstrated significantly higher peak serum concentration after a morning dose compared to an evening dose. Also, the mean oral clearance and volume of distribution after the morning dose was lower comparing to the night dose [62]. Zolmitriptan and sumatriptan attenuate the circadian phase-shifting effects of light by 5HT1B receptor activation in the SCN in Syrian hamsters and without causing phase shifts on their own [63]. These findings are likely applicable to 
human subjects as well, and triptans might play a role in reducing photosensitivity and minimizing circadian disturbances caused by light exposure.

Circadian rhythm can also affect toxicity of a medication resulting in toxic levels or prolonged exposure to the drug. For example CYP-mediated metabolism plays an important role in acetaminophen toxicity, which is more severe in the evening, shown that Cyp2e1 enzyme has a diurnal expression, higher in the nighttime [64]. In the animal model experiments it was demonstrated that plasma concentration and toxicity of Tramadol varies with circadian rhythm and is highest at night [65]. Thus, knowing the circadian specifics of analgesics metabolism helps to maximize its efficacy and minimize side effects and toxicity of the treatments. Morphine and its metabolite M3G were shown to have circadian variations throughout a 24-hour cycle, being 54\% higher in the middle of the dark phase compared to the start of the light phase [66]. Epidural analgesia duration was noted to be longer in diurnal period compared with nocturnal, similar pattern was reported in oral local anesthesia by mepivacaine lasting longer at 15:00 compared to night and early morning $[67,68]$. With more and more information available regarding the specifics of chronotherapy for various medications, we will be able to apply abortive therapy in optimized chronotherapeutic regiments

\section{Preventive Migraine Management and Circadian Rhythm}

There are three major lines of preventive migraine therapy: anti-hypertensive drugs, tricyclic antidepressants and antiepileptic dugs (AEDs), each of the groups demonstrating circadian variations in efficacy. Verapamil, a calcium channel blocker, effective in treatment of migraines and cluster headaches is known to alter circadian rhythm of blood pressure being most effective if administered bedtime, which results in the high concentration of the drug in the morning [69, 70]. Propranolol was also found to have circadian variations in pharmacokinetics, demonstrating the shortest half-life when taken at 8:00 and the longest when taken at 20:00. Time to peak was found to be the shortest when taken at 08:00 [71]. Finally, propranolol was shown to shift circadian phase itself [72].

There is a growing amount of evidence toward circadian rhythmicity of epileptic seizures, which could be related to cortical excitability during sleep and wakefulness and increase of seizure probability in the morning following sleep deprivation [73]. Migraine and epilepsy have some overlap, both recognized as originating from electrical disturbances in the brain and rhythmicity on a circadian time scale, sometimes referred to as a singular phenomenon, "migralepsy" [74]. Chronotherapeutic dosing of AEDs, such as phenytoin and carbamazepine shows improved response of diurnally active epileptic patterns and reduced toxicity when administered at 20:00 in comparison to conventional administration of AEDs [75]. Depakote is often used as a preventive migraine medication and might play a role in affecting circadian rhythms by disrupting oscillatory expression of genes which play a role in regulation of those rhythms [60].Growing evidence of AEDs being more effective in chronotherapeutic administration for treatment of seizures and the overlap of migraine with epilepsy treatment, as well as overlap in pathophysiology of migraine and epilepsy, allow us to suggest that more evidence will arise towards efficacy of chronotherapy in treatment of chronic migraines.

Chronopharmalogical profiles of several antidepressants, were suggested to have circadian rhythmicity when studied in animal model. Antidepressant effect of tricyclic antidepressant (TCA) 
imipramine in mouse model was observed to be highest in the evening in tail region, though plasma and brain levels of the drug were slightly higher in the morning [76]. Circadian changes of pharmacokinetics of amitriptyline in rats demonstrated highest bioavailability in the dark phase around 4:00 with acrophases for clearance appearing between 19:00 and 21:00 [77]. TCAs are mainly inactivated by CYP450 enzyme in the liver; thus the variation of the serum concentration of the TCAs can be explained by circadian regulation of the CYP450 [78]. Small heterodimer partner (SHP) is a circadian gene, which seems to regulate liver circadian clock machinery and CYPs [14]. There is not enough data on circadian fluctuations of TCA pharmacokinetics in human subjects in the literature; thus it could be an area of further investigation.

Onabotulinum toxin (BTA) injections have been used since the early 2000's for chronic migraine prevention with great results [79]. Our group demonstrated that chronic migraine patients with a stable circadian sleep/wake routine benefited the most from BTA injections administered in the afternoon. Although not tested directly, pharmacokinetic research on BTA variants offered some clues as to why this effect might be happening. BTA1 is the only subtype commercially available for chronic migraine treatment and it spreads throughout the tissue better than BTA2 [80, 81]. BTA3 subtype has slightly decreased potency compared to BTA1, likely related to a less efficient SNAP-25 hydrolysis and LC stability [82]. Therefore, hypothesized circadian variability of BTA1 efficiency is likely related to difference in cell entry and/or toxin spread during different circadian phases [80]. We have also demonstrated that discomfort associated with BTA injections is significantly less in the afternoon compared to patients injected in the morning, thus likely improving compliance [83].

\section{Discussion}

Circadian rhythms regulate physiological processes, and play an important role in pain modulation and migraine. The fact that the same neuroanatomical structures are involved in sleepwake regulation and migraine pathogenesis lends itself to a number of possible treatment optimizations. On the molecular level, core circadian genes were found to be activated in pain modulation and sensitivity to pain and development of small-molecule clock modulators should eventually allow for testing of these in migraine management. There is more research to come on molecular mechanisms of migraine and its relationship to circadian genes. Currently available research of chronotherapy is a good foundation to build on for the future research and application of chronotherapy for chronic migraine. Improved understanding of the association between circadian rhythms and migraine variants will lead to significant changes in clinical practice and patient outcomes. Timing of therapeutic interventions, ranging from oral intake of medications to injections administrations should be optimized based on comprehension of these biological systems in standard of care protocols that should include preferred therapeutic windows.

\section{Author Contributions}

Yulia Salamatova: have been involved in writing the manuscript, revising the manuscript, performing the literature search. Andreja Packard: have been involved in drafting the manuscript, revising the manuscript, have given the final approval of the version to be published. 


\section{Competing Interests}

The authors have declared that no competing interests exist.

\section{References}

1. Panda S, Hogenesch JB, Kay SA. Circadian rhythms from flies to human. Nature. 2002; 417: 329335.

2. Eckel-Mahan K, Sassone-Corsi P. Metabolism and the circadian clock converge. Physiol Rev. 2013; 93: 107-135.

3. Gachon F, Nagoshi E, Brown SA, Ripperger J, Schibler U. The mammalian circadian timing system: From gene expression to physiology. Chromosoma. 2004; 113: 103-112.

4. Harrisingh MC, Nitabach MN. Integrating circadian timekeeping with cellular physiology. Science. 2008; 320: 879-880.

5. Murphy PJ, Campbell SS. Physiology of the circadian system in animals and humans. J Clin Neurophysiol. 1996; 13: 2-16.

6. Rijo-Ferreira F, Takahashi JS. Genomics of circadian rhythms in health and disease. Genome Med. 2019; 11: 1-16.

7. Serin $\mathrm{Y}$, Tek NA. Effect of circadian rhythm on metabolic processes and the regulation of energy balance. Ann Nutr Metab. 2019; 74: 322-330.

8. Ralph MR, Foster RG, Davis FC, Menaker M. Transplanted suprachiasmatic nucleus determines circadian period. Science. 1990; 247: 975-978.

9. Berson DM, Dunn FA, Takao M. Phototransduction by retinal ganglion cells that set the circadian clock. Science. 2002; 295: 1070-1073.

10. Gekakis N, Staknis D, Nguyen HB, Davis FC, Wilsbacher LD, King DP, et al. Role of the CLOCK protein in the mammalian circadian mechanism. Science. 1998; 280: 1564-1569.

11. Preitner N, Damiola F, Zakany J, Duboule D, Albrecht U, Schibler U. The orphan nuclear receptor REV-ERB $\alpha$ controls circadian transcription within the positive limb of the mammalian circadian oscillator. Cell. 2002; 110: 251-260.

12. Cox KH, Takahashi JS. Circadian clock genes and the transcriptional architecture of the clock mechanism. J Mol Endocrinol. 2019; 63: R93-R102.

13. Ripperger JA, Schibler U. Rhythmic CLOCK-BMAL1 binding to multiple E-box motifs drives circadian Dbp transcription and chromatin transitions. Nat Genet. 2006; 38: 369-374.

14. Zhang R, Lahens NF, Ballance HI, Hughes ME, Hogenesch JB. A circadian gene expression atlas in mammals: Implications for biology and medicine. Proc Natl Acad Sci U S A. 2014; 111: 1621916224.

15. Aviram J, Shochat T, Pud D. Pain perception in healthy young men is modified by time-of-day and is modality dependent. Pain Med. 2015; 16: 1137-1144.

16. Koltyn KF, Focht BC, Ancker JM, Pasley J. Experimentally induced pain perception in men and women in the morning and evening. Int J Neurosci. 1999; 98: 1-11.

17. Strian F, Lautenbacher S, Galfe G, Hölzl R. Diurnal variations in pain perception and thermal sensitivity. Pain. 1989; 36: 125-131.

18. Fox AW, Davis RL. Migraine chronobiology. Headache. 1998; 38: 436-441.

19. Baksa D, Gecse K, Kumar S, Toth Z, Gal Z, Gonda X, et al. Circadian variation of migraine attack onset: A review of clinical studies. BioMed Res Int. 2019; 2019: 1-9. 
20. Van Oosterhout W, Van Someren E, Schoonman G, Louter M, Lammers G, Ferrari MD, et al. Chronotypes and circadian timing in migraine. Cephalalgia. 2018; 38: 617-625.

21. Segal JP, Tresidder KA, Bhatt C, Gilron I, Ghasemlou N. Circadian control of pain and neuroinflammation. J Neurosci Res. 2018; 96: 1002-1020.

22. Bernstein C, Burstein R. Sensitization of the trigeminovascular pathway: Perspective and implications to migraine pathophysiology. J Clin Neurol. 2012; 8: 89-99.

23. Wright Jr KP, Drake AL, Frey DJ, Fleshner M, Desouza CA, Gronfier C, et al. Influence of sleep deprivation and circadian misalignment on cortisol, inflammatory markers, and cytokine balance. Brain Behav Immun. 2015; 47: 24-34.

24. Martami F, Jahromi SR, Togha M, Ghorbani Z, Seifishahpar M, Saidpour A. The serum level of inflammatory markers in chronic and episodic migraine: A case-control study. Neurol Sci. 2018; 39: 1741-1749.

25. Xia T, Cui Y, Qian Y, Chu S, Song J, Gu X, et al. Regulation of the NR2B-CREB-CRTC1 signaling pathway contributes to circadian pain in murine model of chronic constriction injury. Anesth Analg. 2016; 122: 542-552.

26. Morioka N, Saeki M, Sugimoto T, Higuchi T, Zhang FF, Nakamura Y, et al. Downregulation of the spinal dorsal horn clock gene Per1 expression leads to mechanical hypersensitivity via c-jun Nterminal kinase and CCL2 production in mice. Mol Cell Neurosci. 2016; 72: 72-83.

27. Saper CB, Scammell TE, Lu J. Hypothalamic regulation of sleep and circadian rhythms. Nature. 2005; 437: 1257-1263.

28. Akerman S, Goadsby PJ. Neuronal $\mathrm{PAC}_{1}$ receptors mediate delayed activation and sensitization of trigeminocervical neurons: Relevance to migraine. Sci Transl Med. 2015; 7: 308ra157.

29. Schytz HW, Birk S, Wienecke T, Kruuse C, Olesen J, Ashina M. PACAP38 induces migraine-like attacks in patients with migraine without aura. Brain. 2009; 132: 16-25.

30. Zagami AS, Edvinsson L, Goadsby PJ. Pituitary adenylate cyclase activating polypeptide and migraine. Ann Clin Transl Neurol. 2014; 1: 1036-1040.

31. Hauge AW, Kirchmann M, Olesen J. Characterization of consistent triggers of migraine with aura. Cephalalgia. 2011; 31: 416-438.

32. Ong JC, Taylor HL, Park M, Burgess HJ, Fox RS, Snyder S, et al. Can circadian dysregulation exacerbate migraines? Headache. 2018; 58: 1040-1051.

33. Knippschild U, Gocht A, Wolff S, Huber N, Löhler J, Stöter M. The casein kinase 1 family: Participation in multiple cellular processes in eukaryotes. Cell Signal. 2005; 17: 675-689.

34. Brennan K, Bates EA, Shapiro RE, Zyuzin J, Hallows WC, Huang Y, et al. Casein kinase i $\delta$ mutations in familial migraine and advanced sleep phase. Sci Transl Med. 2013; 5: 183ra156.

35. Hay KM, Mortimer MJ, Barker DC, Debney LM, Good PA. 1044 Women with migraine: The effect of environmental stimuli. Headache. 1994; 34: 166-168.

36. Liampas I, Siokas V, Brotis A, Vikelis M, Dardiotis E. Endogenous melatonin levels and therapeutic use of exogenous melatonin in migraine: Systematic review and meta-analysis. Headache. 2020; 60: 1273-1299.

37. Bekkelund S, Lilleng $\mathrm{H}$. Impact of extreme light exposure during summer season in an arctic area on patients with migraine and chronic paroxysmal hemicrania (CPH). Cephalalgia. 2006; 26: 1153-1156.

38. Bougea A, Spantideas N, Lyras V, Avramidis T, Thomaidis T. Melatonin $4 \mathrm{mg}$ as prophylactic therapy for primary headaches: A pilot study. Funct Neurol. 2016; 31: 33-37. 
39. Gelfand AA, Goadsby PJ. The role of melatonin in the treatment of primary headache disorders. Headache. 2016; 56: 1257-1266.

40. Gonçalves AL, Ferreira AM, Ribeiro RT, Zukerman E, Cipolla-Neto J, Peres MF. Randomised clinical trial comparing melatonin $3 \mathrm{mg}$, amitriptyline $25 \mathrm{mg}$ and placebo for migraine prevention. J Neurol Neurosurg Psychiatry. 2016; 87: 1127-1132.

41. Anderson G. Integrating pathophysiology in migraine: Role of the gut microbiome and melatonin. Curr Pharm Des. 2019; 25: 3550-3562.

42. Torelli P, Evangelista A, Bini A, Castellini P, Lambru G, Manzoni GC. Fasting headache: A review of the literature and new hypotheses. Headache. 2009; 49: 744-752.

43. Kelman L. The triggers or precipitants of the acute migraine attack. Cephalalgia. 2007; 27: 394402.

44. Schulte LH, Jürgens TP, May A. Photo-, osmo-and phonophobia in the premonitory phase of migraine: Mistaking symptoms for triggers? J Headache Pain. 2015; 16: 1-5.

45. Turner DP, Smitherman TA, Penzien DB, Porter JA, Martin VT, Houle TT. Nighttime snacking, stress, and migraine activity. J Clin Neurosci. 2014; 21: 638-643.

46. Amin FM, Aristeidou S, Baraldi C, Czapinska-Ciepiela EK, Ariadni DD, Di Lenola D, et al. The association between migraine and physical exercise. J Headache Pain. 2018; 19: 1-9.

47. Youngstedt SD, Elliott JA, Kripke DF. Human circadian phase-response curves for exercise. J Physiol. 2019; 597: 2253-2268.

48. Pilati L, Battaglia G, Di Stefano V, Di Marco S, Torrente A, Raieli V, et al. Migraine and sport in a physically active population of students: Results of a cross-sectional study. Headache. 2020; 60: 2330-2339.

49. Hindiyeh NA, Krusz JC, Cowan RP. Does exercise make migraines worse and tension type headaches better? Curr Pain Headache Rep. 2013; 17: 380.

50. Dong D, Yang D, Lin L, Wang S, Wu B. Circadian rhythm in pharmacokinetics and its relevance to chronotherapy. Biochem Pharmacol. 2020; 178: 114045.

51. Davidson AJ, Castañón-Cervantes O, Stephan FK. Daily oscillations in liver function: Diurnal vs circadian rhythmicity. Liver Int. 2004; 24: 179-186.

52. Ede MC. Circadian rhythms of drug effectiveness and toxicity. Clin Pharmacol Ther. 1973; 14: 925-935.

53. Firsov D, Bonny O. Circadian regulation of renal function. Kidney Int. 2010; 78: 640-645.

54. Konturek P, Brzozowski T, Konturek S. Gut clock: Implication of circadian rhythms in the gastrointestinal tract. J Physiol Pharmacol. 2011; 62: 139-150.

55. Reinberg A, Smolensky M. Circadian changes of drug disposition in man. Clin Pharmacokinet. 1982; 7: 401-420.

56. Cao QR, Kim TW, Choi JS, Lee BJ. Circadian variations in the pharmacokinetics, tissue distribution and urinary excretion of nifedipine after a single oral administration to rats. Biopharm Drug Dispos. 2005; 26: 427-437.

57. Clench J, Reinberg A, Dziewanowska Z, Ghata J, Smolensky M. Circadian changes in the bioavailability and effects of indomethacin in healthy subjects. Eur J Clin Pharmacol. 1981; 20: 359-369.

58. Ohdo S, Nakano S, Ogawa N. Chronopharmacokinetics of valproic acid following constant-rate administration in mice. Chronobiol Int. 1991; 8: 35-43. 
59. Yoshiyama Y, Nakano S, Ogawa N. Chronopharmacokinetic study of valproic acid in man: Comparison of oral and rectal administration. J Clin Pharmacol. 1989; 29: 1048-1052.

60. Griggs CA, Malm SW, Jaime-Frias R, Smith CL. Valproic acid disrupts the oscillatory expression of core circadian rhythm transcription factors. Toxicol Appl Pharmacol. 2018; 339: 110-120.

61. McLellan TM, Caldwell JA, Lieberman HR. A review of caffeine's effects on cognitive, physical and occupational performance. Neurosci Biobehav Rev. 2016; 71: 294-312.

62. Poondru S, Devaraj R, Boinpally RR, Yamasani MR. Chronopharmacokinetics of sumatriptan in healthy human subjects. J Pharm Pharmacol. 2000; 52: 1085-1090.

63. Basu P, le N, Wensel AL, Baskerville JD, Smith VM, Antle MC. Triptans attenuate circadian responses to light. Eur J Neurosci. 2015; 42: 2489-2495.

64. Matsunaga N. Dosing time based on molecular mechanism of biological clock of hepatic drug metabolic enzyme. Yakugaku Zasshi. 2009; 129: 1357-1365.

65. Liu XP, Song JG. Chronopharmacology of tramadol in mice. Yao Xue Xue Bao. 2001; 36: 561-564.

66. Kervezee L, Hartman R, van den Berg DJ, Meijer JH, de Lange EC. Diurnal variation in the pharmacokinetics and brain distribution of morphine and its major metabolite. Eur J Pharm Sci. 2017; 109: S132-S139.

67. Debon R, Chassard D, Duflo F, Boselli E, Bryssine B, Allaouchiche B. Chronobiology of epidural ropivacaine: Variations in the duration of action related to the hour of administration. J Am Soc Anesthesiol. 2002; 96: 542-545.

68. Pöllmann L. Circadian changes in the duration of local anaesthesia. Int J Oral Surg. 1982; 11: 3639.

69. Burish MJ, Chen Z, Yoo SH. Emerging relevance of circadian rhythms in headaches and neuropathic pain. Acta Physiol. 2019; 225: e13161.

70. Smolensky MH, Portaluppi F. Chronopharmacology and chronotherapy of cardiovascular medications: Relevance to prevention and treatment of coronary heart disease. Am Heart J. 1999; 137: S14-S24.

71. Langner B, Lemmer B. Circadian changes in the pharmacokinetics and cardiovascular effects of oral propranolol in healthy subjects. Eur J Clin Pharmacol. 1988; 33: 619-624.

72. Shimamoto H, Shimamoto $Y$, Sakata S. Influences of propranolol and atenolol on the circadian rhythm of heart rate in elderly patients with essential hypertension. Clin Sci. 1990; 78: 403-407.

73. Khan S, Nobili L, Khatami R, Loddenkemper T, Cajochen C, Dijk DJ, et al. Circadian rhythm and epilepsy. Lancet Neurol. 2018; 17: 1098-1108.

74. Nye BL, Thadani VM. Migraine and epilepsy: Review of the literature. Headache. 2015; 55: 359380.

75. Yegnanarayan R, Mahesh SD, Sangle S. Chronotherapeutic dose schedule of phenytoin and carbamazepine in epileptic patients. Chronobiol Int. 2006; 23: 1035-1046.

76. Kawai H, Iwadate R, Ishibashi T, Kudo N, Kawashima Y, Mitsumoto A. Antidepressants with different mechanisms of action show different chronopharmacological profiles in the tail suspension test in mice. Chronobiol Int. 2019; 36: 1194-1207.

77. Rutkowska A, Piekoszewski W, Brandys J. Chronopharmacokinetics of amitriptyline in rats. Biopharm Drug Dispos. 1999; 20: 117-124.

78. Gillman P. Tricyclic antidepressant pharmacology and therapeutic drug interactions updated. Br J Clin Pharmacol. 2007; 151: 737-748. 
79. Aurora SK, Gawel M, Brandes JL, Pokta S, VanDenburgh AM, Group BNAEMS. Botulinum toxin type a prophylactic treatment of episodic migraine: A randomized, double-blind, placebocontrolled exploratory study. Headache. 2007; 47: 486-499.

80. Packard A, Arciniegas AA, Smotherman C. Effectiveness of preventive onabotulinumtoxin A injections for migraine headaches is dependent on the circadian time of administration. Chronobiol Int. 2021; 38: 576-583.

81. Torii Y, Kiyota N, Sugimoto N, Mori Y, Goto Y, Harakawa T, et al. Comparison of effects of botulinum toxin subtype $\mathrm{A} 1$ and $\mathrm{A} 2$ using twitch tension assay and rat grip strength test. Toxicon. 2011; 57: 93-99.

82. Henkel JS, Jacobson M, Tepp W, Pier C, Johnson EA, Barbieri JT. Catalytic properties of botulinum neurotoxin subtypes $A 3$ and $A 4$. Biochemistry. 2009; 48: 2522-2528.

83. Packard A, Smotherman C, Jovanovic N. Effect of circadian rhythm on the pain associated with preventive onabotulinumtoxinA injections for migraines. Chronobiol Int. 2020; 37: 1766-1771.

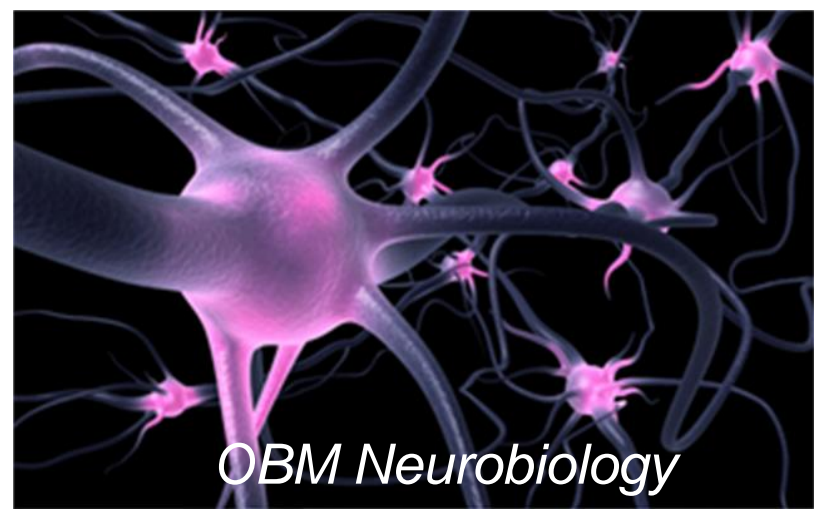

Enjoy OBM Neurobiology by:

1. Submitting a manuscript

2. Joining volunteer reviewer bank

3. Joining Editorial Board

4. Guest editing a special issue

For more details, please visit:

http://www.lidsen.com/journals/neurobiology 